Ashdin Publishing

Journal of Generalized Lie Theory and Applications

Vol. 4 (2010), Article ID G100501, 5 pages

doi:10.4303/jglta/G100501

\title{
Cheban loops
}

\author{
J. D. Phillips ${ }^{a}$ and V. A. Shcherbacov ${ }^{b}$ \\ ${ }^{a}$ Department of Mathematics and Computer Science, Northern Michigan University, \\ Marquette, MI 49855, USA \\ ${ }^{b}$ Institute of Mathematics, Academy of Sciences of Moldova, str. Academiai 5, MD 2028, \\ Chisinau, Moldova
}

E-mails: jophilli@nmu.edu,scerb@math.md

\begin{abstract}
Left Cheban loops are loops that satisfy the identity $x(x y \cdot z)=y x \cdot x z$. Right Cheban loops satisfy the mirror identity $(z \cdot y x) x=z x \cdot x y$. Loops that are both left and right Cheban are called Cheban loops. Cheban loops can also be characterized as those loops that satisfy the identity $x(x y \cdot z)=(y \cdot z x) x$. These loops were introduced by A. M. Cheban. Here we initiate a study of their structural properties. Left Cheban loops are left conjugacy closed. Cheban loops are weak inverse property, power associative, conjugacy closed loops; they are centrally nilpotent of class at most two.
\end{abstract}

2000 MSC: 20N05

\section{Introduction}

In [15], Osborn showed that if $L$ is a weak inverse property loop all of whose loop isotopes also have the weak inverse property, then the nucleus of $L$ is normal and the quotient of $L$ by its nucleus is Moufang (definitions are given in the next section). He showed further that $L$ must satisfy the identity $x(y z \cdot x)=((x \cdot y x) / x) \cdot z x$. In [1], Basarab coined the term Osborn loop to describe those loops that satisfy this identity.

The variety of Osborn loops contains as subvarieties two of the most important classes of loops - the Moufang loops and the conjugacy closed loops [12]. One of the most important open problems in loop theory is to determine whether or not all loop isotopes of an arbitrary Osborn loop are themselves Osborn.

In [2], Basarab showed that the quotient of a conjugacy closed loop by its nucleus is an abelian group. Also in [1], he showed that weak inverse property Osborn loops are characterized by the identity $x(y z \cdot x)=(((1 / y) \cdot(1 / x)) \backslash 1) \cdot z x$. He called these loops generalized Moufang loops.

In [5], Cheban investigated the structure of two varieties of loops: those loops that satisfy the identity $x(x y \cdot z)=y x \cdot x z$ and those loops that satisfy the identity $x(x y \cdot z)=(y \cdot z x) x$. We call these the left Cheban identity and the Cheban identity, respectively. Cheban showed that loops satisfying his second identity are generalized Moufang loops and that each of them has an abelian group as an image, which is not surprising in the light of Theorem 3.10 below. He also gave an example of a loop satisfying his first identity but that was not Moufang.

As we will see, Cheban's two varieties have many other strong structural properties; they are also intimately related to conjugacy closed loops.

Our investigations were aided by the automated reasoning tool Prover9 and the finite model builder Mace4 [13]. We have translated most of these computer generated proofs into 
more "human friendly" form and included them in this paper. A few of the proofs, though, are quite long, and we have left them untranslated. However, each of these is posted on the first author's website [18], and is clearly referenced in the proofs in this paper. We note that it is a common practice to publish complicated untranslated Prover9 proofs [14, 19]. This is mathematically sound since the program can be made to output a simple proof object, which can be independently verified by a short lisp program.

\section{Definitions}

A loop $(Q, \cdot)$ is a set $Q$ with a binary operation - such that (i) for each $x \in Q$, the left translation $L(x): Q \rightarrow Q ; y \mapsto x y$ and the right translation $R(x): Q \rightarrow Q ; y \mapsto y x$ are bijections, and (ii) there exists $1 \in Q$ satisfying $1 \cdot x=x \cdot 1=x$ for all $x \in Q$. Standard references for the theory of loops are $[3,4,16]$.

A Moufang loop is a loop that satisfies $x y \cdot z x=(x \cdot y z) x$. A flexible loop satisfies $x \cdot y x=x y \cdot x$. The left alternative property, denoted by LAP, is given by $x \cdot x y=x x \cdot y$. The RAP is the mirror identity of the LAP.

The left nucleus of a loop $Q$ is given by $\mathrm{N}_{\lambda}(Q)=\{a: a \cdot x y=a x \cdot y, \forall x, y \in L\}$. The middle nucleus, $\mathrm{N}_{\mu}(Q)$, and the right nucleus, $\mathrm{N}_{\rho}(Q)$, are defined analogously. The nucleus, then, is given by $\mathrm{N}(Q)=\mathrm{N}_{\lambda}(Q) \cap \mathrm{N}_{\mu}(Q) \cap \mathrm{N}_{\rho}(Q)$. The commutant of $Q$ is given by $\mathrm{C}(Q)=$ $\{c: \forall x \in Q, c x=x c\}$. The center is the normal subloop given by $\mathrm{Z}(Q)=\mathrm{N}(Q) \cap \mathrm{C}(Q)$. Now, define $\mathrm{Z}_{0}(Q)=\{1\}$, and $\mathrm{Z}_{i+1}(Q), i \geq 0$, as the preimage of $\mathrm{Z}\left(Q / \mathrm{Z}_{i}(Q)\right)$ under the canonical projection. The loop $Q$ is (centrally) nilpotent of class $n$, written $c \ell(Q)=n$, if $\mathrm{Z}_{n-1}(Q)<\mathrm{Z}_{n}(Q)=Q$.

A loop, $Q$, is left conjugacy closed, denoted by LCC, if its left translations are closed under conjugation by left translations, that is, if $L(x)^{-1} L(y) L(x)$ is itself a left translation for each $x, y \in Q$. This can be expressed equationally as $z \cdot y x=((z y) / z) \cdot z x$. Right conjugacy closed, denoted by RCC, is the mirror identity. A loop $Q$ is conjugacy closed, denoted by CC, if it is both LCC and RCC. The concept of conjugacy closedness was introduced first by Solkis [21] and later independently by Goodaire and Robinson [9]. In the intervening years, a great deal has been discovered about their structural properties, see, e.g., [7].

An especially prominent role in the analysis of CC-loops is assumed by the weak inverse property elements, or WIP elements; these are elements $c$ such that for every $x$ in the loop we have $c(x c)^{\rho}=x^{\rho}$, where $\rho$ is the unary operation that gives the right inverse of each element $y$ in the loop, that is $y y^{\rho}=1$. A loop is power associative if subloops generated by singletons are, in fact, groups. Power associative conjugacy closed loops have especially strong structural properties [10]; this variety is denoted by PACC.

A triple of bijections $(f, g, h)$ from a loop $Q_{1}$ to a loop $Q_{2}$ is called a (loop) isotopism if

$$
f(x) \cdot g(y)=h(x \cdot y)
$$

for every $x, y$ in $Q_{1}$. Note that $f$ is an isomorphism if and only if $(f, f, f)$ is an isotopism.

Finally, an identity $\alpha=\beta$ is of Bol-Moufang type if (i) the only operation in $\alpha, \beta$ is ·, (ii) the same 3 variables appear on both sides, in the same order, (iii) one of the variables appears twice on both sides, (iv) the remaining two variables appear once on both sides. For instance, the Moufang law given above, $x y \cdot z x=(x \cdot y z) x$, is an identity of Bol-Moufang type. The varieties of loops classified by a single identity of Bol-Moufang type were classified in [20]. The Cheban identities are not identities of Bol-Moufang type, since the variables do not appear in the same order on both sides of the equal sign. But they do satisfy the 
other conditions of the definition. The varieties of loops classified by a single identity of this generalized Bol-Moufang type are classified in [6].

\section{Theorems}

Theorem 3.1. A loop, $Q$, is left Cheban if and only if it is $L C C$ and $R(x)^{2}=L(x)^{2}$ for all $x \in Q$.

Proof. In the left Cheban identity, $x(x y \cdot z)=y x \cdot x z$, let $z=1$ obtain $R(x)^{2}=L(x)^{2}$. Using this, we obtain $(x \cdot x y) / x=(y x \cdot x) / x=y x$. This in turn yields $(x \backslash y) x=(x \cdot x(x \backslash y)) / x=$ $(x y) / x$. Finally, this, together with the left Cheban law, gives $((x y) / x) \cdot x z=((x \backslash y) x) \cdot x z=$ $x(x(x \backslash y) \cdot z)=x \cdot y z$.

For the converse, first rearrange the LCC law to get

$$
((x y) / x) \backslash(x \cdot y z)=x z .
$$

Next, use $R(x)^{2}=L(x)^{2}$ to get $(x \cdot x y) / x=y x$. Now set $y=x \backslash z$ in this to get

$$
(x z) / x=(x \backslash z) x .
$$

Combine (3.1) and (3.2) to get $((x \backslash y) x) \backslash(x \cdot y z)=x z$. Now use this to get $(x y) \backslash(y(y x$. $z))=((y \backslash(y x)) y) \backslash(y(y x \cdot z))=y z$. Finally, multiply both sides of this by $x y$ to obtain $x y \cdot y z=(x y) \cdot((x y) \backslash(y(y x \cdot z)))=y(y x \cdot z)$.

Remark 3.2. The variety of WIP PACC-loops can be axiomatized, in the variety of loops, by the following two identities: $(x y \cdot x) \cdot x z=x \cdot(y x \cdot x) z$ and $z x \cdot(x \cdot y x)=(z(x \cdot x y)) \cdot x$ [17]. Left Cheban loops satisfy the first of these two identities, as the reader may check.

Theorem 3.3. Let $Q$ be a left Cheban loop. Then $\mathrm{N}_{\lambda}(Q)=\mathrm{N}_{\mu}(Q) \unlhd Q$ and $\mathrm{C}(Q) \leq \mathrm{N}(Q)$. Moreover, if $a \in \mathrm{N}_{\lambda}(Q)$, then $a^{2} \in \mathrm{Z}(Q)$.

Proof. The fact that $\mathrm{N}_{\lambda}(Q)=\mathrm{N}_{\mu}(Q) \unlhd Q$ follows from [8, Proposition 2.7], since any left Cheban loop is LCC-loop by Theorem 3.1.

Let $a$ be a left nuclear element. Then $x \cdot a y=(x / a) a \cdot a y=a((a \cdot x / a) y)=(a(a \cdot x / a)) y=$ $((x / a \cdot a) a) y=x a \cdot y$, that is, $a$ is a middle nuclear element. The converse is left to the reader.

Now, let $b$ be a commutant element. We have $x \cdot y b=x \cdot b y=(x / b) b \cdot b y=b(b(x / b) \cdot y)=$ $b((x / b) b \cdot y)=b \cdot x y=x y \cdot b$. Thus, $b$ is a right nuclear element. For the proof that $b$ is also middle, and hence left, nuclear, see [18]. Finally, let $a$ be a left nuclear element. Then, since $R(a)^{2}=L(a)^{2}$, we have $x \cdot a a=x a \cdot a=a \cdot a x=a a \cdot x$, that is, $a^{2}$ is a commutant element.

Example 3.4. Here is a left Cheban loop in which 1 is nuclear but not central. This example is of minimal order.

$\begin{array}{llllllll}0 & 1 & 2 & 3 & 4 & 5 & 6 & 7 \\ 1 & 0 & 3 & 2 & 5 & 4 & 7 & 6 \\ 2 & 4 & 0 & 6 & 1 & 7 & 3 & 5 \\ 3 & 5 & 1 & 7 & 0 & 6 & 2 & 4 \\ 4 & 2 & 6 & 0 & 7 & 1 & 5 & 3 \\ 5 & 3 & 7 & 1 & 6 & 0 & 4 & 2 \\ 6 & 7 & 4 & 5 & 2 & 3 & 0 & 1 \\ 7 & 6 & 5 & 4 & 3 & 2 & 1 & 0\end{array}$


Theorem 3.5. Let $Q$ be a left Cheban loop. If $c$ is a WIP element, then $c^{2}$ is central. Moreover, for every $x \in Q, x^{2}$ is a WIP element, and hence, $x^{4}$ is central.

Proof. See [18].

Lemma 3.6. A loop is Cheban if and only if it is both left and right Cheban.

By the left Cheban law and the right Cheban law, respectively, we have $x(x y \cdot z)=$ $y x \cdot x z=(y \cdot z x) x$. For the converse, see [18].

Lemma 3.7. Let $Q$ be a left Cheban loop. If $Q$ is a WIP loop or if $R\left(x^{2}\right)=L\left(x^{2}\right)$ for all $x \in Q$, then $Q$ is, in fact, a Cheban loop.

Proof. See [18].

In preparation for the next theorem, recall that an extra loop is a loop that satisfies the identity $x(y \cdot z x)=(x y \cdot z) x$. Extra loops are conjugacy closed; in extra loops, squares are nuclear [11].

Lemma 3.8. Let $Q$ be a left Cheban loop. If $Q$ is either flexible or satisfies the RAP, then $Q$ is, in fact, an extra loop (and, obviously, a Cheban loop).

Proof. See [18].

Lemma 3.9. In a cancelative CC-groupoid, the following two conditions are equivalent:

(1) $R\left(x^{2}\right)=L\left(x^{2}\right)$ for all $x$,

(2) $R(x)^{2}=L(x)^{2}$ for all $x$.

The straightforward proof is left to the reader.

Theorem 3.10. A loop, $Q$, is Cheban if and only if it is conjugacy closed and satisfies $R\left(x^{2}\right)=L\left(x^{2}\right)$ for all $x \in Q$.

Proof. Combine Theorem 3.1, Lemma 3.6, and Lemma 3.8.

Theorem 3.11. Cheban loops are WIP PACC-loops. Moreover, they are centrally nilpotent of class at most 2.

Proof. The fact that Cheban loops are WIP PACC-loops is straightforward and left to the reader. By Basarab's theorem, in CC loops the commutant is contained in the center. Also by Theorem 3.10, squares are contained in the commutant, hence they are also contained in the center. So the factor of a Cheban loop by its center has exponent 2 and is, hence, an abelian group, which finishes the proof of the theorem.

Remark 3.12. A WIP PACC-loop of nilpotency class 2 need not be Cheban, as evidenced by any nonabelian group of odd order and nilpotency class 2 (since in this case, $R\left(x^{2}\right)=L\left(x^{2}\right)$ will not hold for all $x$ ).

Remark 3.13. If $L$ is a left Cheban, right Cheban, or Cheban loop in which every element is either a square or an involution, then $L$ is an abelian group, as the reader may easily check. 


\section{References}

[1] A. S. Basarab. A class of WIP loops (Russian). Mat. Issled., 2 (1967), 3-24.

[2] A. S. Basarab. A class of LK-loops (Russian). Mat. Issled., no. 120, Bin. i $n$-arnye Kvazigruppy (1991), 3-7.

[3] V. D. Belousov. Foundations of the Theory of Quasigroups and Loops (Russian), Izdat. "Nauka", Moscow, 1967.

[4] R. H. Bruck. A Survey of Binary Systems, Springer-Verlag, Berlin, 1958.

[5] A. M. Cheban. Loops with identities of length four and of rank three. II (Russian). In "General Algebra and Discrete Geometry", pp. 117-120, 164, "Shtiintsa", Kishinev, Moldova, 1980.

[6] B. Coté, B. Harvill, M. Huhn, and A. Kirchman. Classification of loops of generalized BolMoufang type, submitted to Quasigroups Related Systems.

[7] P. Csörgö. Extending the structural homomorphism of LCC loops. Comment. Math. Univ. Carolin., 46 (2005), 385-389.

[8] A. Drápal. On multiplication groups of left conjugacy closed loops. Comment. Math. Univ. Carolin., 45 (2004), 223-236.

[9] E. G. Goodaire and D. A. Robinson. A class of loops which are isomorphic to all loop isotopes. Canad. J. Math., 34 (1982), 662-672.

[10] M. K. Kinyon and K. Kunen. Power-associative, conjugacy closed loops. J. Algebra, 304 (2006), 679-711.

[11] M. K. Kinyon and K. Kunen. The structure of extra loops. Quasigroups Related Systems, 12 (2004), 39-60.

[12] M. K. Kinyon, K. Kunen, and J. D. Phillips, On Osborn loops. Unpublished manuscript.

[13] W. W. McCune. Prover9, Automated Reasoning Software, Argonne National Laboratory, http://www.cs.unm.edu/ mccune/mace4/, 2005.

[14] W. W. McCune and R. Padmanabhan. Automated Deduction in Equational Logic and Cubic Curves. Lecture Notes in Computer Science, Vol. 1095. Lecture Notes in Artificial Intelligence. Springer-Verlag, Berlin, 1996.

[15] J. M. Osborn. Loops with the weak inverse property. Pacific J. Math., 10 (1960), 295-304.

[16] H. O. Pflugfelder. Quasigroups and Loops: Introduction. Sigma Series in Pure Mathematics, Vol. 7. Heldermann Verlag, Berlin, 1990.

[17] J. D. Phillips. A short basis for the variety of WIP PACC-loops. Quasigroups Related Systems, 14 (2006), 73-80.

[18] J. D. Phillips. http://euclid.nmu.edu/ jophilli/paper-supplements.html

[19] J. D. Phillips. The Moufang laws, global and local. J. Algebra Appl., 8 (2009), 477-492.

[20] J. D. Phillips and P. Vojtěchovský. The varieties of loops of Bol-Moufang type. Algebra Universalis, 54 (2005), 259-271.

[21] L. R. Soukis. The special loops (Russian). In "Questions of the Theory of Quasigroups and Loops". pp. 122-131, Redakc.-Izdat. Otdel Akad. Nauk Moldav. SSR, Kishinev, 1970.

Received February 1, 2010

Revised April 11, 2010 